\title{
A rare situation in acute rheumatic carditis: Involvement of all four valves
}

\author{
Osman Güvenç ${ }^{1}$, Derya Çimen ${ }^{2}$ \\ ${ }^{1}$ Division of Pediatric Cardiology, Gynecologic and Pediatric Hospital, Batman; ${ }^{2}$ Division of Pediatric Cardiology, Medova \\ Hospital, Konya, Turkey. E-mail: osmanguvenc1977@gmail.com \\ Receive: 3rd January 2017, Revised: 11th February 2017, Accepted: 12th February 2017
}

\begin{abstract}
SUMMARY: Güvenç O, Çimen D. A rare situation in acute rheumatic carditis: Involvement of all four valves. Turk J Pediatr 2017; 59: 497-500.

Acute rheumatic fever continues to be an important health problem, especially in countries that are socioeconomically underdeveloped. Carditis, which develops in approximately half of the patients, is responsible for both earlystage mortality as well as late-stage surgical treatment due to heart valve insufficiency or stenosis. The most frequent and severe valve involvement is with the mitral valve, while the aortic valve has the second highest incidence of involvement. Pulmonary and tricuspid valves are rarely involved. The literature cites a few adult cases in which all four valves are affected by rheumatic carditis; however, to the best of our knowledge, there have been no acute-stage rheumatic carditis pediatric cases reported. This article presents a 13-year-old male patient of Syrian origin who escaped to Turkey from the war in his country, and who was in the acute stage of rheumatic carditis in which all four valves were involved.
\end{abstract}

Key words: acute rheumatic fever, four valves, childhood, quadrivalvular.

Acute rheumatic fever (ARF) has been the most frequent cause of cardiac disease in underdeveloped and developing countries. Unfortunately, it continues to pose a significant problem today. Every year, 300,000 people develop rheumatic cardiac disease globally. ${ }^{1,2}$ In non-cardiac involvement, when no sequelae remain, such as arthritis and chorea, the prognosis is good. However, in approximately $30-70 \%$ of the cases, cardiac involvement is seen and can determine the prognosis of the disease, especially if pancarditis develops.

The mitral valve is most frequently affected, followed by involvement of the aortic valve. Cases in which the pulmonary and tricuspid valves are also affected are rarely seen. While mild valvular insufficiencies may completely disappear in time, moderate and severe valvular involvements, as well as valves injured due to repeated attacks, may later require surgical valve repair or valve replacement. ${ }^{3}$ In this article, we present a 13-year-old patient diagnosed with active-stage ARF in which all four valves are involved. This rarely seen case is discussed here in reference to existing literature.

\section{Case Report}

This 13-year-old male patient of Syrian origin presented to our clinic complaining of pain first in his right knee and then his left elbow, which had been present for three days. The family reported that the patient had not had any throat infections in the past month and none of his family members had cardiac rheumatism. The patient's weight and height were within normal range for his age; his systemic blood pressure was 105/65 mmHg; and his pulse was $110 / \mathrm{min}$. The patient had arthritis in his right knee and left elbow, and his precordium was hyperactive.

The examination identified a pansystolic murmur at a severity of $4 / 6$. The most significant sign during auscultation at the apex was a diastolic murmur at a severity of $2 / 4$, better auscultated in the precordium with thrills upon palpation. The femoral pulses could be received bilaterally. No signs of cardiac failure, hepatosplenomegaly, or lymphadenopathy were detected in the patient; the examination results for other systems were normal. His sinus rhythm was 107 beats/min during electrocardiographic 
examination no dysrhythmias or atrioventricular blocks were seen. The telecardiographic examination identified the cardiothoracic ratio as 0.53 (Fig. 1). As seen in the transthoracic echocardiography (TTE) scan, the mitral leaflets had become thickened and mitral valve prolapse was present; the mitral valve was observed to have significant insufficiency, the aortic valve had moderate insufficiency, the pulmonary valve had mild-moderate, and the tricuspid valve had severe insufficiency (Fig. 2). The left cardiac chambers were dilated (left ventricle end-diastolic diameter of $50 \mathrm{~mm}, \mathrm{z}$ score + 2 ), and systolic functions were measured as normal (EF 68\%, FS 44\%). The patient did not have any echocardiographic signs that would suggest pulmonary hypertension (PHT).

The laboratory results showed a leukocyte count of $13,600 / \mathrm{mm}^{3}$, a C-reactive protein (CRP) of $108 \mathrm{mg} / \mathrm{L}$ (normal range $0-5$ ), a sedimentation

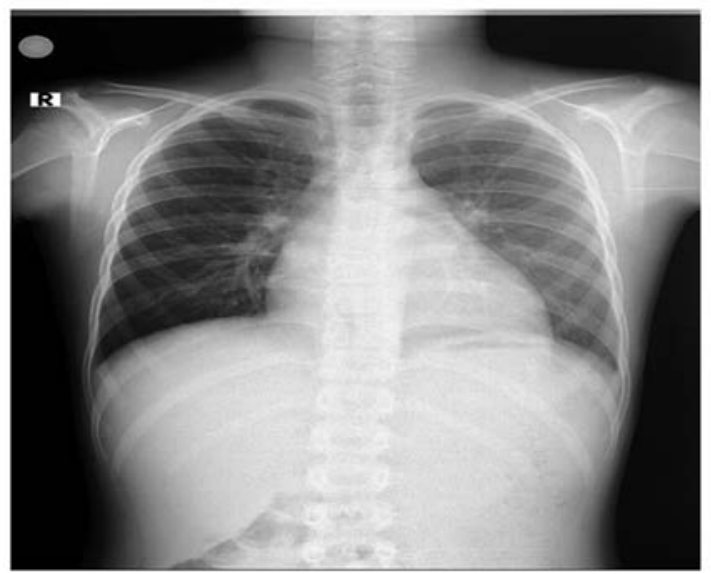

Fig. 1. Cardiomegaly is observed during the telecardiographic examination. rate of $103 \mathrm{~mm} /$ hour, and the antistreptolysin O (ASO) titer was 560 Todd units (normal range 0-250). The other hematological and biochemical values were within normal range. The patient was diagnosed with acute rheumatic carditis; he was hospitalized and put on absolute bed rest.

Treatment was started with oral prednisolone, captopril, furosemide, lansoprazole and prophylaxis with intramuscular benzathine penicillin. The patient's arthritis disappeared on the second day of treatment; his tachycardia regressed; and his acute phase reactants were measured as normal on day-10. The echocardiographic controls showed a significant decrease in pulmonary and tricuspid valve regurgitation, which persisted at a mild rate, while there were no changes in the severities of aortic and mitral valve insufficiency. The patient's prednisolone dose was tapered after four weeks, and an anti-inflammatory dose of acetylsalicylic acid was added to the treatment. $\mathrm{He}$ was discharged with recommendations to continue his medical treatment at home. The family was informed about the disease and their consent was taken to publish this case.

\section{Discussion}

In the acute stage of rheumatic fever, endocardial involvement occurs and valve insufficiency develops. During the late stage, fibrosis develops that frequently involves the mitral valve, which results in valvular stenosis. ${ }^{1}$ Endocardial involvement initially results in edema, macrophage and fibroblast infiltration in the valves, and small thrombi, termed verrucous vegetation, develop on the closure surfaces
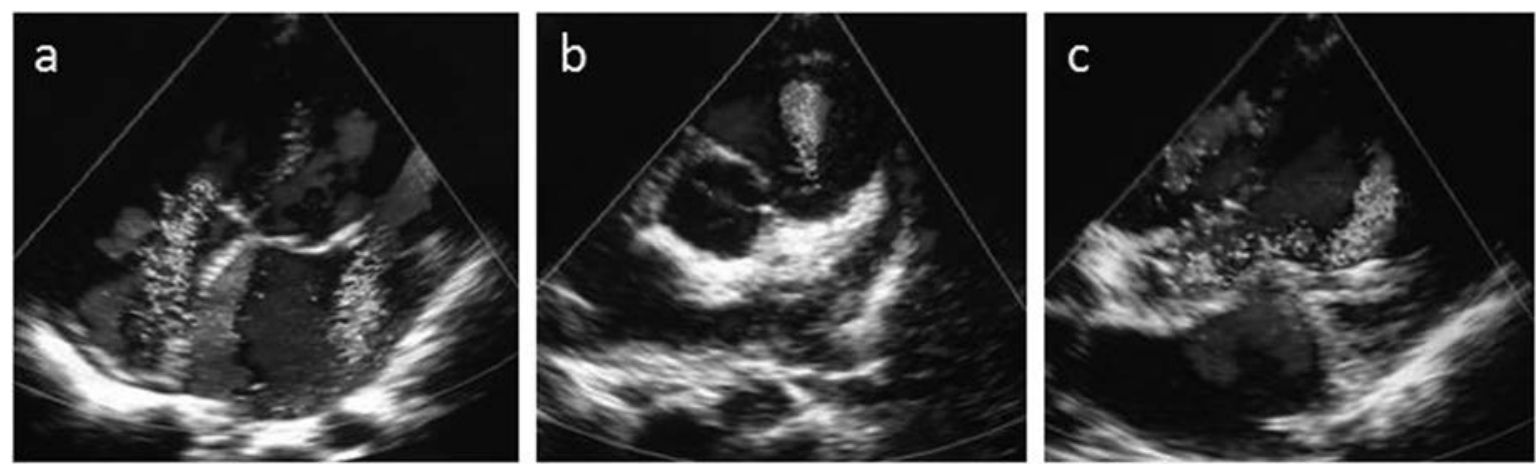

Fig. 2. (a) Insufficiency of the mitral and tricuspid valves is seen in the apical four-chamber view during the transthoracic echocardiographic examination. During the transthoracic echocardiographic examination; (b) Pulmonary valve insufficiency on the parasternal short axis is observed, (c) Aortic valve insufficiency on the parasternal long axis is observed. 
of the leaflets. Annular dilatation develops in the valves, and coaptation deteriorates in the chordae tendineae in the mitral valve due to distention, eventually resulting in valvular insufficiency. The chordae tendineae may also rupture. Over time, inflammation improves with fibrosis, the valve thickens, and stenosis occurs. ${ }^{1,2}$ Since the mitral valve closes at a high pressure, it is most often severely affected, while the aortic valve is slightly less affected. Among patients with acute rheumatic carditis, 95\% have mitral valve insufficiency, while $20-25 \%$ have aortic valve insufficiency, which is generally accompanied by mitral valve insufficiency. Aortic valve prolapse is the most frequent cause of aortic valve insufficiency. The more severe the carditis is, the higher the number of valves involved, which negatively affects the disease progress. ${ }^{1,3}$

The right-sided valves are affected by rheumatic cardiac disease, and insufficiency and/or stenosis develop along with significant mitral and/or aortic valve involvement. Tricuspid and pulmonary valves are rarely affected, and their isolated involvement without the involvement of mitral and aortic valves is almost never observed. Tricuspid valve involvement with rheumatic carditis is more frequent than pulmonary valve involvement; however, it is not expected that both valves would be affected at a clinically significant degree ${ }^{4}$. One study determined that $7.7 \%$ of 2497 rheumatic cardiac diseases had valve involvement, with 99\% of those involving the mitral valve. However, another study determined that the tricuspid valve was involved in $9 \%$ of 788 ARF patients with significant mitral valve injury, suggesting that tricuspid valve involvement among rheumatic cardiac disease patients was not rare. This finding was overlooked, however, because it attracted less attention than the aortic and mitral valve involvements. Yet another study indicated that the reason was rheumatic cardiac disease in less than $10 \%$ of patients with significant tricuspid insufficiency. Patients who previously had rheumatic carditis may later develop significant tricuspid stenosis in adulthood. ${ }^{5-7}$ In our case, we observed that all four of our patient's valves were affected during the acute carditis stage, and the mitral valve was the most severely affected.

These patients may develop pulmonary venous congestion and pulmonary edema secondary to severe mitral valve insufficiency. In conclusion, secondary PHT develops and this situation may manifest itself as right ventricular dysfunction, right cardiac failure and significant tricuspid and pulmonary insufficiency. Whether the tricuspid and pulmonary valve insufficiency of our case was due to the primary valve involvement or to the PHT, which occurred due to severe mitral valve insufficiency, is subject to controversy. ${ }^{1,4}$ There is no other PHT finding other than tricuspid valve insufficiency on the TTE examination of the patient at the first admission. Tricuspid and pulmonary valve insufficiency have been significantly reduced by treatment with steroids, parallel to the rapid recovery in clinical and acute phase reactants. Therefore, an organic valve entrapment due to ARF is considered rather than a functional valve entrapment.

There are few publications in the literature reporting the involvement of all four valves with ARF disease and it is known that this is a rather rare situation. These case reports are generally adult patients who are known to have had ARF in their childhood and developed valvular stenosis later in their lives. To the best of our knowledge, there are no rheumatic cardiac patients reported to have had all four valves involved during acute carditis in childhood. One report cites the case of a thirty-five-year-old patient who had four valves involved due to repeated ARF attacks in childhood. His mitral, aortic, and pulmonary valves were replaced, and the tricuspid valve underwent annuloplasty. ${ }^{8-10}$ Today, the number of patients with multiplevalve involvement or severe valve injury has decreased since echocardiographic imaging has become common; hence, valve involvements are noticed in more patients as compared to the past, and proper treatment and penicillin prophylaxis are started at an early phase. ${ }^{11-13}$

Studies have also found that disease progression or permanent cardiac injury does not change with anti-inflammatory treatment. However, this treatment enables the acute phase reactants to regress more rapidly, and the number of patients undergoing surgical treatment due to valve disease has decreased. Surgical intervention during an acute attack of the disease is not preferred, although valvuloplasty or valve replacement may be required as long- 
term $^{3}$ follow-up in symptomatic patients with severe valve insufficiency, progressive left ventricular expansion, or with limited exercise capacity. Considering that our patient is a war refugee, and taking his living conditions into account, it is possible that he might have previously suffered from silent carditis and that the picture he presented to us might be a recurrence of the disease.

In conclusion, mitral valves and aortic valves are commonly affected secondary to rheumatic carditis in children; however, it should be remembered that tricuspid and pulmonary valves may also be affected. It is not always easy to determine whether tricuspid and pulmonary valve insufficiency stems from rheumatic carditis or from another underlying reason.

\section{REFERENCES}

1. Remenyi B, Carapetis J. Acute rheumatic fever and chronic rheumatic disease. In: Da Cruz EM, Ivy D, Jaggers J (ed). Pediatric and Congenital Cardiology, Cardiac Surgery and Intensive Care. London: SpringerVerlag, 2014: 2329-2350.

2. Wilson SE, Chinyere UC, Queennette D. Childhood acquired heart disease in Nigeria: An echocardiographic study from three centres. Afr Health Sci 2014; 14: 609-616.

3. Park MK. Obstructive Lesions. In: Park MK. Park's Pediatric Cardiology for Practitioners. Philadelphia: Elsevier Saunders, 2014: 184-205.
4. Sultan FA, Moustafa SE, Tajik J, et al. Rheumatic tricuspid valve disease: an evidence-based systematic overview. J Heart Valve Dis 2010; 19: 374-382.

5. Talwar S, Jayanthkumar HV, Sharma G, Kumar AS. Quadrivalvular rheumatic heart disease. Int J Cardiol 2006; 106: 117-118.

6. Shiran A, Najjar R, Adawi S, Aronson D. Risk factors for progression of functional tricuspid regurgitation. Am J Cardiol 2014; 113: 995-1000.

7. Katz MG, Schachner A, Harpaz D, Kravtsov V, Rozenman Y, Sasson L. Quadrivalvular heart disease: Transition from congenital pulmonary stenosis to rheumatic disease. J Heart Valve Dis 2007; 16: 96-100.

8. Bandin MA, Vargas-Barron J, Keirns C, RomeroCardenas A, Villegas M, Buendia A. Echocardiographic diagnosis of rheumatic cardiopathy affecting all four cardiac valves. Am Hear J 1990; 120: 1004-1007.

9. Krishnamoorthy KM. Rheumatic stenosis of all four valves. Texas-Heart Inst J 2002; 29: 224-225.

10. Gupta SK, Abraham AK, Mani GK, Cherian KM, Moorthy JS, Bhaskaran K. Quadrivalvular rheumatic heart disease. Jpn Heart J 1987; 28: 507-514.

11. Gialloreto O, Aerichide N, Allard PP. Stenotic involvement of all four heart valves: Report of three cases. Am J Cardiol 1961; 7: 865-873.

12. Kumar N, Rasheed K, Gallo R, Al-Halees Z, Duran $\mathrm{CM}$. Rheumatic involvement of all four heart valvespreoperative echocardiographic diagnosis and successful surgical management. Eur J Cardiothorac Surg 1995; 9: 713-714

13. Kumar AS, Iyer KS, Chopra P. Quadrivalvular heart disease. Int J Cardiol 1985; 7: 66-69. 